\title{
INVESTIGATIONS ON THE EFFECT OF BORON CARBIDE AND GRAPHITE REINFORCEMENTS DURING SPARK EROSIIN MACHINING OF ALUMINIUM 5052 HYBRID COMPOSITE
}

\author{
Nikhil Zachariah Sabu, Rahul C, Sudarsan R, Gautham Raaj Kumar K \\ UG Students, Department of Mechanical Engineering, Kumaraguru College of Technology, \\ Coimbatore - 641049, Tamilnadu, India
}

\begin{abstract}
Automobile, Marine and Aerospace industries are eager on introducing Aluminium metal matrix composites due to their excellent mechanical properties which makes reduction in the weight of the component. In the present study, based on the literature review, the fabrication of Aluminium metal matrix composite (ALUMINIUM/ $\mathrm{B}_{4}$ C/GRAPHITE) is discussed and spark erosion machining process is carried out to investigate the machining properties exhibited by the hybrid composite.
\end{abstract}

\section{INTRODUCTION}

Reinforcements introduced to metal matrix composites are known for their inherent properties like corrosion resistance, wear resistance and machinability. This study deals with the investigation of the effect of adding Boron Carbide (B4C) and graphite (Gr) reinforcements in aluminum alloy (AA 5052) hybrid composite material over the spark erosion machining performance. The composite material is fabricated using stir casting process. The boron carbide and graphite particles are introduced in various weight proportion combinations to aluminum alloy (AA 5052) and spark erosion machining was carried out on the composite specimens. Pulse Time ON and OFF, Machining time, Material Removal Rate and Tool Wear are the process parameters taken into account.

\section{PROBLEM DEFINITION AND SOLUTION}

\section{A. Problem Statement}

The study is to investigate the effect of Boron Carbide and Graphite reinforcements in aluminum 5052 hybrid composite material over the spark erosion machining performance. This study helps to determine the change in the machining time and the material removing rate of the specimen during the machining process. This study aims on improving the machining properties of the alloy.
The machining time and material removal rate of the work piece made from aluminum, boron carbide and graphite is measured for different mixtures of different weight proportions. With the observations made from the study we will be able find the mixture with the best mechanical properties when compared to others.

\section{CONSTRUCTIONAL SETUP}

This experiment requires a stir casting machine for the fabrication of the hybrid composite. It is the most simplest and cost effective method of liquid state fabrication. The addition of two alloys with Aluminium5052 is done through stir casting process. In this process AA5052 is held in crucible at 700 degree Celsius. When aluminium gets fully liquefied, finely powdered granules of Boron Carbide and Graphite is added to the molten matrix. After all alloys are bonded thoroughly, it is allowed to cool and taken as a mould piece out. This process is conducted in three different weight proportions as mentioned below. All the three weight proportions are then machined using spark erosion process and the values are compared to determine which particular proportion compartively has better machining properties.

\section{SNON}

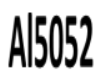

\section{Graphite}

Boron Carbide
1.

2.

3. 3448
$16 \mathrm{~g}$

$16 \mathrm{~g}$

$16 \mathrm{~g}$

\section{8}

328

408

\section{B. Problem Solution}


International Journal of Engineering Applied Sciences and Technology, 2020

Vol. 5, Issue 5, ISSN No. 2455-2143, Pages 209-212

Published Online September 2020 in IJEAST (http://www.ijeast.com)

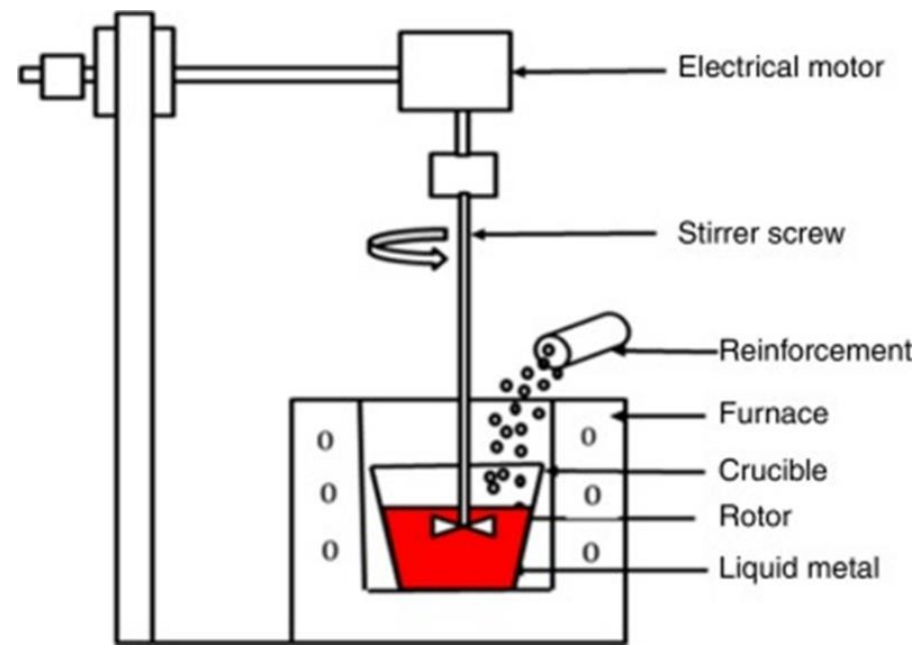

Figure 1. Stir Casting

The prepared material is to be machined using Electric Discharge Machine (EDM). In a spark erosion machining unit, an electrode to work piece relationship is maintained in a liquid medium (di-electric fluid). The dielectric fluid functions as a conductor and coolant during the procedure. When the cutting gap is sufficiently small, the fluid ionizes allowing electric discharges to occur. These electric discharges occur at regular intervals and such cycles take place about 250,000 times a second. Pulse Time On \& Off, Machining Time, Material Removal Rate and Tool Wear are measured.

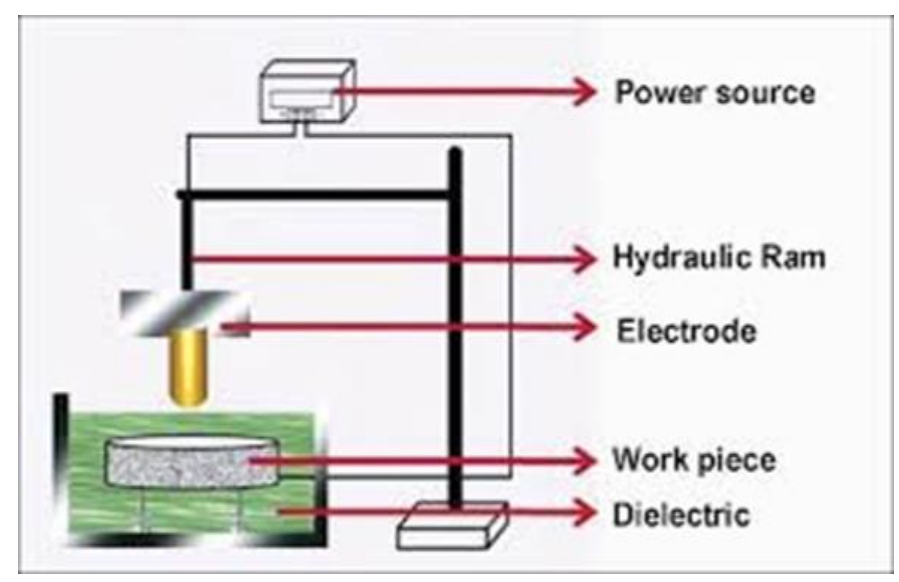

Figure 2. Spark Erosion Machining

\section{WORKING}

Aluminium alloy 5052 is reinforced with different weight proportions of boron carbide and graphite. The required composite material is achieved by stir casting method. Stir casting method is a liquid state method of composite materials fabrication, in which a dispersed phase is mixed with a molten matrix metal by means of mechanical stirring. The aluminium alloy is molten at
700 degree Celsius in the crucible. Finely powdered granules of the reinforcements are introduced into the crucible and stirred mechanically for almost 15 minutes and is allowed to cool in a mould.

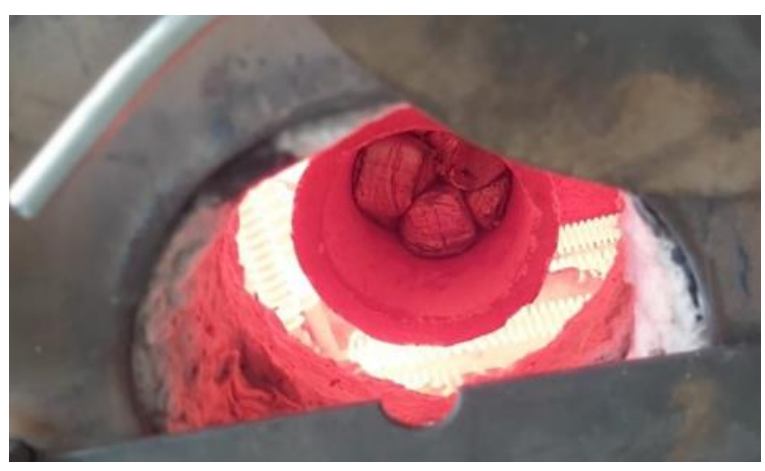

Figure 3. Casting

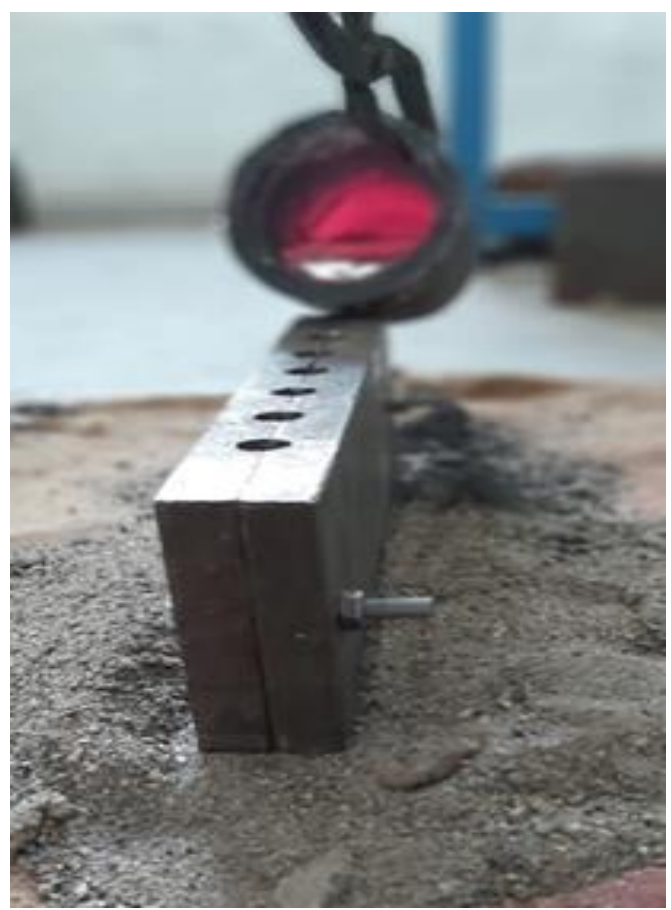

Figure 4. Transfer of cast to mould

In a spark erosion machining unit, an electrode to work piece relationship is maintained in a liquid medium. The electrode is usually made of zinc and is shaped into the negative form of the desired shape by CAD/CAM or milling. The di electric fluid used in this process in deionized water. The work piece is the metal which is to be shaped into the desired form. The electrode moves towards and away from the work piece assisted by a hydraulic ram connected to it during the process. Once the machining process is done the material removal rate and machining time of the specimen is noted. Three different specimens of different weight percentages are used for this experiment. In the first trial $4 \%$ of graphite, $92 \%$ of aluminum and $4 \%$ of boron carbide is 
used, in the second trial $4 \%$ of graphite, $88 \%$ of aluminum and $8 \%$ of boron carbide is used and in the third trial $4 \%$ of graphite, $90 \%$ of aluminum and $10 \%$ of boron carbide is used. The machining time and material removal rate of these three trials is then calculated. The observations is then compared and the trial work piece with best qualities is chosen.

\section{OBSERVATIONS}

The as-cast AA 5052 alloy, when examined with 1000x magnification revealed uniform structural integrity in the microstructure. The EDS spectrum proved the absence of significant impurities thereby indicating the purity of the ascast alloy sample. The microstructure of the as-cast aluminium alloy AA 5052 revealed the presence of a solid solution of the matrix alloy throughout the sample0[Fig 5]. Also, $\alpha$-aluminium dendritic structure was observed from the evaluation, which had grown during the accelerated cooling of the aluminium melt, during solidification process. The presence of free carbon was detected from the recorded EDS spectrum due to the addition of $8 \%$ and $10 \%$ by weight of WC particles and $4 \%$ by $10 \%$ weight of graphite particles in the aluminium alloy matrix. This has proved the uniform distribution of graphite particles in the composite. Moreover, the microstructure confirmed the uniform dispersion of the WC particles and this uniform dispersion do not depend on the concentration of the particulates in the samples.

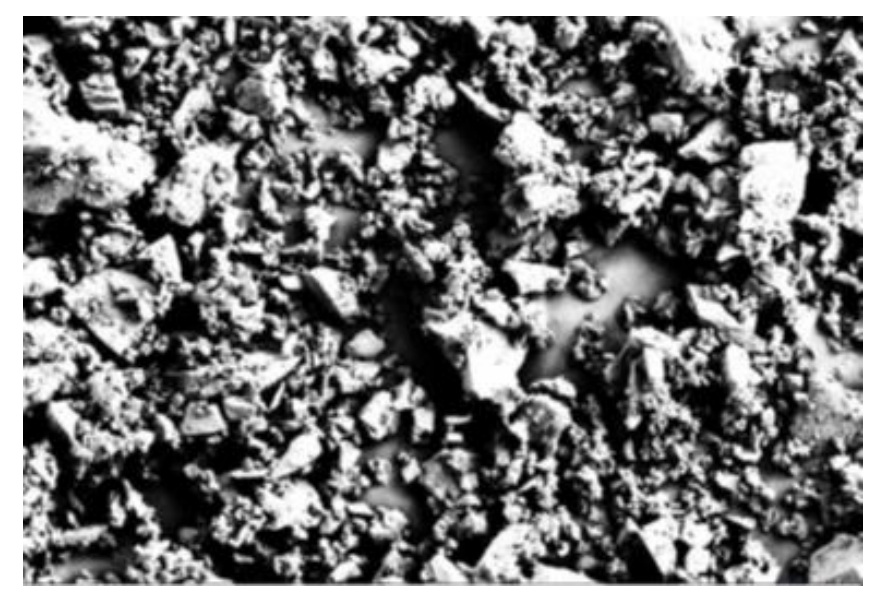

Figure 5. Microstructure of the composite

\section{TABULATION}

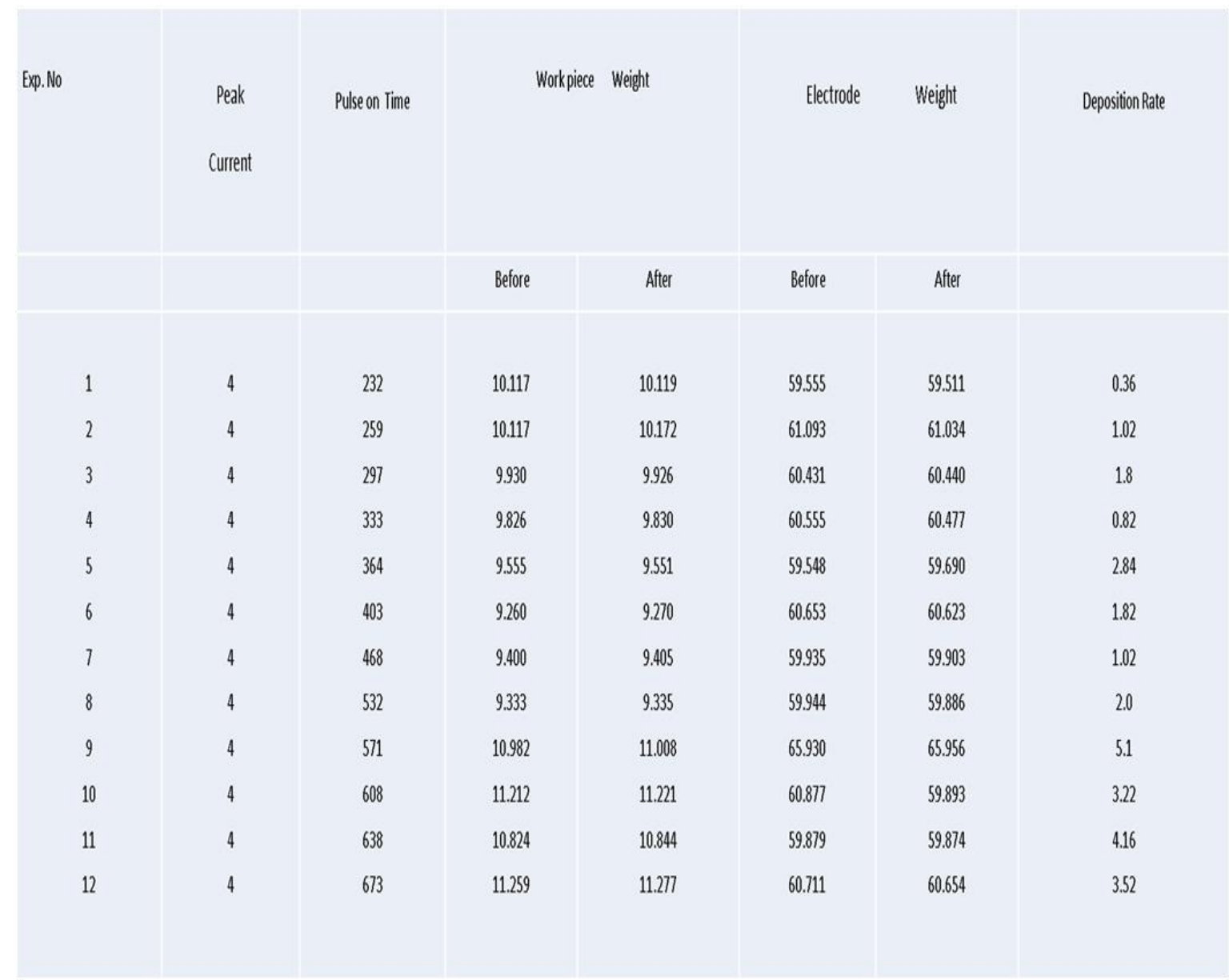




\section{CONCLUSION AND FUTURE SCOPE}

\section{A. Conclusion}

- The improvement in mechanical properties attained due to the addition of Boron Carbide and graphite in AA 5052 enable this $\mathrm{Al} / \mathrm{B}_{4} \mathrm{C} /$ graphite hybrid composite to be a potential material in marine and offshore structural applications.

- Boron Carbide improves the strength of the specimen while graphite improves the thermal and electrical conductivity of the specimen.

- It was also found from the investigations that $10 \%$ by weight of Boron Carbide and $4 \%$ by weight of graphite particles could be considered as the best composition in achieving improvement in the density, micro hardness, impact strength, tensile strength and peak elongation of the AA 5052 hybrid composites.

\section{B. Future scope}

- Due to the exceptional corrosion resistance against sea water, it is used for failure sensitive large marine structures like the tanks of liquefied Natural Gas tankers, Ships etc.

- Aluminium alloy composite is widely desired in the manufacture of electrical enclosures, fuel tanks, aircraft tubes, refrigerators and marine vessels.

- This alloy composite is also a good alternative for the conventional material that is used for the production of hydraulic tubes.

\section{REFERENCES}

1. Rao Ramo S., Padmanabhan G., Fabrication and mechanical properties of aluminium boron carbide composites.

2. Ranganathan, Madhankumar, Saravanan, Karthikeyan, Murali S., Optimization and Characterization of A15052/ Sic Metal Matrix Composite.

3. Gore Abhay S., Patel Nilesh G., Wire Electro Discharging Machining of Metal Matrix Composites.

4. Weingärtnera E., Kustera F., Wegenera D. Modeling and simulation of electrical discharge machining.

5. Jeswani M. L., Roughness and wear characteristics of spark-eroded surfaces.

6. Muthuramalingam T.,Influence of Tool Electrode Properties on Machinability in Spark Erosion Machining.
7. Ko GunYoung, Effect of differential speed rolling strain on microstructure and mechanical properties of nanostructured $5052 \mathrm{Al}$ alloy.

8. Roh Jae-Hun, Seo Jeong-Jin, Hong Sung-Tae A., Kim Moon-Jo C., Heung Nam Han, Roth John T., The mechanical behavior of 5052-H32 aluminum alloys under a pulsed electric current.

9. Das Sandeep, Raman Kumar Randhir, Devarani N., Joshi Shrikrishna N., Surface Alloying of Titanium Di-boride and Silicon Carbide on Aluminium $\mathrm{Al}$ 5052 using Electric Discharge Processing.

10. Jacob Ebenezer D. S., Velmurugan C., Wins Leo Dev K., Investigations on the Effect of Boron Carbide and Graphite Reinforcements During Spark Erosion Machining of Aluminium 5052 Hybrid Composite.

\section{Acknowledgement}

We hereby acknowledge Mr. S Prabhu, Assistant Professor, Department of Mechanical Engineering, Kumaraguru College Of Technology and Mr. Pradeep, Assistant Professor, Department of Mechanical Engineering, Kumaraguru College Of Technology who helped us put the basic platform for this novel design. 